\title{
Dynamical masses for the nearest brown dwarf binary: $\varepsilon$ Indi Ba,b
}

\author{
C. V. Cardoso ${ }^{1}$, M. J. McCaughrean ${ }^{2,1}$, R. R. King ${ }^{1}$, L. M. Close ${ }^{3}$, \\ R.-D. Scholz ${ }^{4}$, R. Lenzen ${ }^{5}$ W. Brandner ${ }^{5}$, N. Lodieu $^{6}$, H. Zinnecker ${ }^{4}$, \\ R. Koehler ${ }^{7}$ and Q. M. Konopacky ${ }^{8}$
}

${ }^{1}$ University of Exeter, ${ }^{2}$ ESA, ${ }^{3}$ Steward Observatory, ${ }^{4}$ Astrophysikalisches Institut Potsdam, ${ }^{5}$ Max-Planck-Institut für Astronomie, ${ }^{6}$ Instituto de Astrofísica de Canarias, ${ }^{7}$ Landessternwarte Zentrum für Astronomie Heidelberg, ${ }^{8} \mathrm{UCLA}$,

Binary brown dwarfs are important because their dynamical masses can be determined in a model-independent way. If a main sequence star is also involved, the age and metallicity for the system can be determined, making it possible to break the sub-stellar mass-age degeneracy. The most suitable benchmark system for intermediate age $\mathrm{T}$ dwarfs is $\varepsilon$ Indi Ba,b, two $\mathrm{T}$ dwarfs (spectral types T1 and T6; McCaughrean et al. (2004)) orbiting a K4.5V star, $\varepsilon$ Indi A, at a projected separation of $1460 \mathrm{AU}$. At a distance of $3.6224 \mathrm{pc}$ (HIPPARCOS distance to $\varepsilon$ Indi A; van Leeuwen (2007)), these are the closest brown dwarfs to the Earth, and thus both components are bright and the system is well-resolved. The system has been monitored astrometrically with NACO and FORS2 on the VLT since June 2004 and August 2005, respectively, in order to determine the system and individual masses independent of evolutionary models. We have obtained a preliminary system mass of $121 \pm 1 \mathrm{M}_{\mathrm{ju}}$. We have also analysed optical/near-IR spectra (0.6-5.0 $\mu \mathrm{m}$ at a resolution up to $\mathrm{R} \sim 5000$; King et al. (2009)) allowing us to determine bolometric luminosities, compare and calibrate evolutionary and atmospheric models of $\mathrm{T}$ dwarfs at an age of $4-8 \mathrm{Gyr}$.

Acknowledgements: This work is funded by the EC FP6 Marie Curie RTN CONSTELLATION: MRTN-CT-2006-035890.
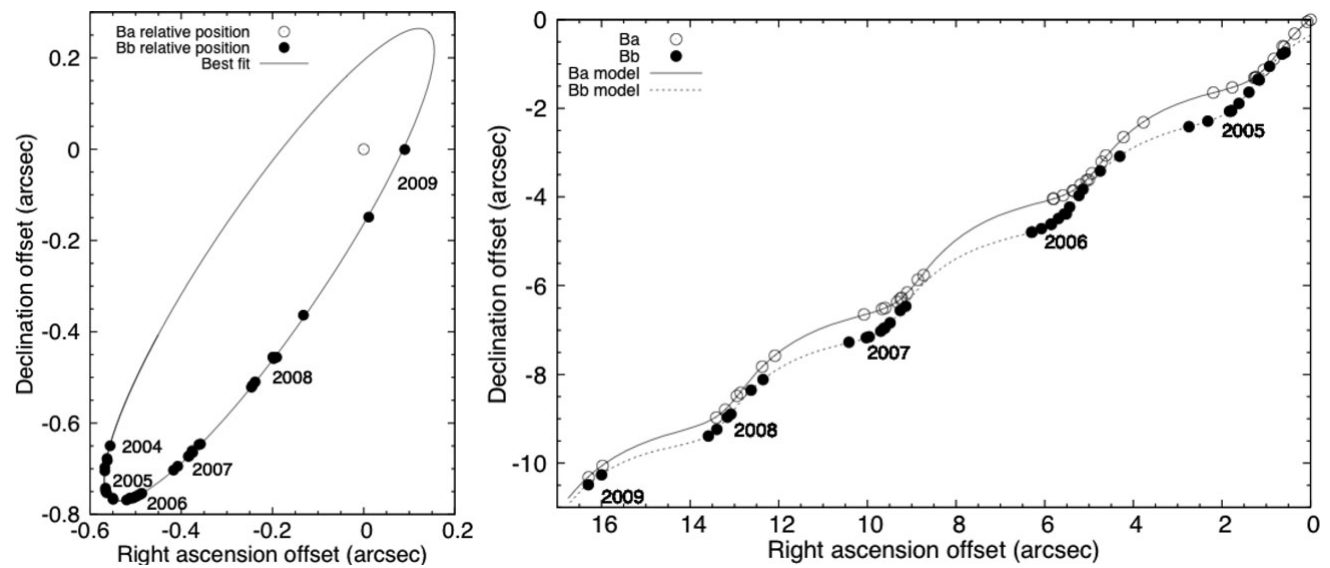

Figure 1. Left: Relative motion (NACO). Right: Absolute motion (FORS2).

\section{References}

McCaughrean, M. J., Close, L. M., Scholz, R.-D., Lenzen, R., et al. 2004, A\&A 413, 1029

van Leeuwen, F. 2007, A\& A 474, 653

King, R. R., McCaughrean, M. J., Homeier, D., Allard, F. et al. 2009, A\&A in press 